\title{
Generalized fractional integral inequalities of Hermite-Hadamard type for harmonically convex functions
}

\author{
Dafang Zhao ${ }^{1,2}$, Muhammad Aamir Ali ${ }^{3 *}$, Artion Kashuri ${ }^{4}$ and Hüseyin Budak ${ }^{5}$
}

\author{
"Correspondence: \\ mahr.muhammad.aamir@gmail.com \\ 3 Jiangsu Key Laboratory for NSLSCS, \\ School of Mathematical Sciences, \\ Nanjing Normal University, Nanjing, \\ China \\ Full list of author information is \\ available at the end of the article
}

\begin{abstract}
In this paper, we establish inequalities of Hermite-Hadamard type for harmonically convex functions using a generalized fractional integral. The results of our paper are an extension of previously obtained results (İşcan in Hacet. J. Math. Stat. 43(6):935-942, 2014 and İşcan and Wu in Appl. Math. Comput. 238:237-244, 2014). We also discuss some special cases for our main results and obtain new inequalities of Hermite-Hadamard type.
\end{abstract}

MSC: 26D07; 26D10; 26D15; 26B15; 26B25

Keywords: Hermite-Hadamard inequalities; Generalized fractional integral; Harmonically convex functions

\section{Introduction}

The Hermite-Hadamard inequality introduced by Hermite and Hadamard, see [4], and [17, p. 137], is one of the best-established inequalities in the theory of convex analysis with a nice geometrical interpretation and several applications. These inequalities state the following.

If $f: I \rightarrow \mathbb{R}$ is a convex function on the interval $I$ of real numbers and $a, b \in I$ with $a<b$, then

$$
f\left(\frac{a+b}{2}\right) \leq \frac{1}{b-a} \int_{a}^{b} f(x) d x \leq \frac{f(a)+f(b)}{2} .
$$

Both inequalities hold in reverse order if the function $f$ is concave. It is worth mentioning that the Hermite-Hadamard inequality may be regarded as a refinement of the concept of convexity and it follows easily from Jensen's inequality. For more results which generalize, unify and extend the inequalities (1.1), see [5-11, 20-24]) and the references therein.

İşcan [8] gave the following definition of harmonically convex functions.

(c) The Author(s) 2020. This article is licensed under a Creative Commons Attribution 4.0 International License, which permits use, sharing, adaptation, distribution and reproduction in any medium or format, as long as you give appropriate credit to the original author(s) and the source, provide a link to the Creative Commons licence, and indicate if changes were made. The images or other third party material in this article are included in the article's Creative Commons licence, unless indicated otherwise in a credit line to the material. If material is not included in the article's Creative Commons licence and your intended use is not permitted by statutory regulation or exceeds the permitted use, you will need to obtain permission directly from the copyright holder. To view a copy of this licence, visit http://creativecommons.org/licenses/by/4.0/. 
Definition 1 ([8]) A function $f: I \subseteq \mathbb{R} \backslash\{0\} \rightarrow \mathbb{R}$ is said to be a harmonically convex function if the following inequality holds:

$$
f\left(\frac{a b}{t a+(1-t) b}\right) \leq t f(b)+(1-t) f(a)
$$

for all $a, b$ in $I$ and $t$ in $[0,1]$. If the inequality (1.2) holds in the reversed direction then $f$ is called harmonically concave function.

İşcan [8] established the following identity and integral inequalities of HermiteHadamard type for harmonically convex functions.

Theorem 1 ([8]) Let $f: I \subseteq \mathbb{R} \backslash\{0\} \rightarrow \mathbb{R}$ be harmonically convex function and $a, b \in I$ with $a<b$. If $f \in L([a, b])$, then the following double inequality holds:

$$
f\left(\frac{2 a b}{a+b}\right) \leq \frac{a b}{b-a} \int_{a}^{b} \frac{f(x)}{x^{2}} d x \leq \frac{f(a)+f(b)}{2} .
$$

Lemma 1 ([8]) Let $f: I \subseteq \mathbb{R} \backslash\{0\} \rightarrow \mathbb{R}$ be differentiable on $I^{\circ}$ (interior of I) and $a, b \in I$ with $a<b$. Iff $\in L([a, b])$, then the following identity holds:

$$
\begin{aligned}
& \frac{f(a)+f(b)}{2}-\frac{a b}{b-a} \int_{a}^{b} \frac{f(x)}{x^{2}} d x \\
& =\frac{a b(b-a)}{2} \int_{0}^{1} \frac{1-2 t}{(t b+(1-t) a)^{2}} f^{\prime}\left(\frac{a b}{t b+(1-t) a}\right) d t .
\end{aligned}
$$

Theorem 2 ([8]) Let $f: I \subseteq(0, \infty) \rightarrow \mathbb{R}$ be differentiable on $I^{\circ}, a, b \in I$ with $a<b$, and $f^{\prime} \in L([a, b])$. If $\left|f^{\prime}\right|^{q}$ is harmonically convex function on $[a, b]$ for $q \geq 1$, then the following inequality holds:

$$
\left|\frac{f(a)+f(b)}{2}-\frac{a b}{b-a} \int_{a}^{b} \frac{f(x)}{x^{2}} d x\right| \leq \frac{a b(b-a)}{2} \lambda_{1}^{1-\frac{1}{q}}\left[\lambda_{2}\left|f^{\prime}(a)\right|^{q}+\lambda_{3}\left|f^{\prime}(b)\right|^{q}\right]^{\frac{1}{q}},
$$

where

$$
\begin{aligned}
& \lambda_{1}=\frac{1}{a b}-\frac{2}{(b-a)^{2}} \ln \left(\frac{(a+b)^{2}}{4 a b}\right), \\
& \lambda_{2}=\frac{-1}{b(b-a)}+\frac{3 a+b}{(b-a)^{3}} \ln \left(\frac{(a+b)^{2}}{4 a b}\right), \\
& \lambda_{3}=\lambda_{1}-\lambda_{2} .
\end{aligned}
$$

Theorem 3 ([8]) Let $f: I \subseteq(0, \infty) \rightarrow \mathbb{R}$ be differentiable on $I^{\circ}, a, b \in I$ with $a<b$, and $f^{\prime} \in L([a, b])$. If $\left|f^{\prime}\right|^{q}$ is harmonically convex function on $[a, b]$ for $q>1, \frac{1}{p}+\frac{1}{q}=1$, then the following inequality holds:

$$
\begin{aligned}
& \left|\frac{f(a)+f(b)}{2}-\frac{a b}{b-a} \int_{a}^{b} \frac{f(x)}{x^{2}} d x\right| \\
& \quad \leq \frac{a b(b-a)}{2}\left(\frac{1}{p+1}\right)^{\frac{1}{p}}\left(\mu_{1}\left|f^{\prime}(a)\right|^{q}+\mu_{2}\left|f^{\prime}(b)\right|^{q}\right)^{\frac{1}{q}},
\end{aligned}
$$


where

$$
\begin{aligned}
& \mu_{1}=\frac{\left[a^{2-2 q}+b^{1-2 q}[(b-a)(1-2 q)-a]\right]}{2(b-a)^{2}(1-q)(1-2 q)}, \\
& \mu_{2}=\frac{\left[b^{2-2 q}+a^{1-2 q}[(b-a)(1-2 q)+b]\right]}{2(b-a)^{2}(1-q)(1-2 q)} .
\end{aligned}
$$

Now we recall some special functions and an inequality that will be needed in the sequel to establish our main results in this paper.

(a) The Beta function is defined as follows:

$$
\beta(x, y)=\frac{\Gamma(x) \Gamma(y)}{\Gamma(x+y)}=\int_{0}^{1} t^{x-1}(1-t)^{y-1} d t, \quad x, y>0 .
$$

(b) The hypergeometric function is given as

$$
{ }_{2} F_{1}(a, b ; c ; z)=\frac{1}{\beta(b, c-b)} \int_{0}^{1} t^{b-1}(1-t)^{c-b-1}(1-z t)^{-\alpha} d t, \quad c>b>0,|z|<1 .
$$

Lemma 2 For $0<\alpha \leq 1$ and $0 \leq a<b$, we have the following inequality:

$$
\left|b^{\alpha}-a^{\alpha}\right| \leq(b-a)^{\alpha}
$$

İşcan [10] also established the following identity and inequalities of Hermite-Hadamard type for harmonically convex functions via Riemann-Liouville fractional integrals.

Theorem 4 ([10]) Let $f: I \subseteq(0, \infty) \rightarrow \mathbb{R}$ be function such that $f \in L([a, b])$, where $a, b \in I$ with $a<b$. If $f$ is harmonically convex function on $[a, b]$, the following double inequality holds for the fractional integrals:

$$
\begin{aligned}
f\left(\frac{2 a b}{a+b}\right) & \leq \frac{\Gamma(\alpha+1)}{2}\left(\frac{a b}{b-a}\right)^{\alpha}\left\{I_{\frac{1}{a}-}^{\alpha}(f \circ g)\left(\frac{1}{b}\right)+I_{\frac{1}{b}+}^{\alpha}(f \circ g)\left(\frac{1}{a}\right)\right\} \\
& \leq \frac{f(a)+f(b)}{2}
\end{aligned}
$$

where $g(x)=\frac{1}{x}$.

Lemma 3 ([10]) Let $f: I \subseteq(0, \infty) \rightarrow \mathbb{R}$ be a differentiable function on $I^{\circ}$ such that $f^{\prime} \in$ $L([a, b])$, where $a, b \in I$ with $a<b$. Then the following identity holds for the fractional integrals:

$$
I_{f}(g ; \alpha, a, b)=\frac{a b(b-a)}{2} \int_{0}^{1} \frac{\left[t^{\alpha}-(1-t)^{\alpha}\right]}{(t b-(1-t) a)^{2}} f^{\prime}\left(\frac{a b}{t b+(1-t) a}\right) d t
$$

where

$$
I_{f}(g ; \alpha, a, b)=\frac{f(a)+f(b)}{2}-\frac{\Gamma(\alpha+1)}{2}\left(\frac{a b}{b-a}\right)^{\alpha}\left\{I_{\frac{1}{a}-}^{\alpha}(f \circ g)\left(\frac{1}{b}\right)+I_{\frac{1}{b}+}(f \circ g)\left(\frac{1}{a}\right)\right\},
$$

and $g$ is as given in Theorem 4. 
Theorem 5 ([10]) Let $f: I \subseteq(0, \infty) \rightarrow \mathbb{R}$ be a differentiable function on $I^{\circ}$ such that $f^{\prime} \in$ $L([a, b])$, where $a, b \in I$ with $a<b$. If $\left|f^{\prime}\right|^{q}$ is harmonically convex function on $[a, b]$ for some fixed $q \geq 1$, then we have the following inequality for the fractional integrals:

$$
\left|I_{f}(g ; \alpha, a, b)\right| \leq \frac{a b(b-a)}{2} C_{1}^{1-\frac{1}{q}}(\alpha ; a, b)\left(C_{2}(\alpha ; a, b)\left|f^{\prime}(b)\right|^{q}+C_{3}(\alpha ; a, b)\left|f^{\prime}(a)\right|^{q}\right)^{\frac{1}{q}},
$$

where

$$
\begin{aligned}
& C_{1}(\alpha ; a, b)=\frac{b^{-2}}{\alpha+1}\left[{ }_{2} F_{1}\left(2,1 ; \alpha+2 ; 1-\frac{a}{b}\right)+{ }_{2} F_{1}\left(2, \alpha+1 ; \alpha+2 ; 1-\frac{a}{b}\right)\right], \\
& C_{2}(\alpha ; a, b)=\frac{b^{-2}}{\alpha+2}\left[\frac{1}{\alpha+1}{ }_{2} F_{1}\left(2,2 ; \alpha+3 ; 1-\frac{a}{b}\right)+{ }_{2} F_{1}\left(2, \alpha+2 ; \alpha+3 ; 1-\frac{a}{b}\right)\right], \\
& C_{3}(\alpha ; a, b)=\frac{b^{-2}}{\alpha+1}\left[{ }_{2} F_{1}\left(2,1 ; \alpha+3 ; 1-\frac{a}{b}\right)+\frac{1}{\alpha+1}{ }_{2} F_{1}\left(2, \alpha+1 ; \alpha+3 ; 1-\frac{a}{b}\right)\right] .
\end{aligned}
$$

Theorem 6 ([10]) Let $f: I \subseteq(0, \infty) \rightarrow \mathbb{R}$ be a differentiable function on $I^{\circ}$ such that $f^{\prime} \in$ $L([a, b])$, where $a, b \in I$ with $a<b$. If $\left|f^{\prime}\right|^{q}$ is harmonically convex function on $[a, b]$ for some fixed $q \geq 1$, then we have the following inequality for the fractional integrals:

$$
\begin{aligned}
& \left|I_{f}(g ; \alpha, a, b)\right| \\
& \quad \leq \frac{a b(b-a)}{2} C_{1}^{1-\frac{1}{q}}(\alpha ; a, b)\left(C_{2}(\alpha ; a, b)\left|f^{\prime}(b)\right|^{q}+C_{3}(\alpha ; a, b)\left|f^{\prime}(a)\right|^{q}\right)^{\frac{1}{q}},
\end{aligned}
$$

where

$$
\begin{aligned}
C_{1}(\alpha ; a, b)= & \frac{b^{-2}}{\alpha+1}\left[{ }_{2} F_{1}\left(2, \alpha+1 ; \alpha+2 ; 1-\frac{a}{b}\right)-{ }_{2} F_{1}\left(2,1 ; \alpha+2 ; 1-\frac{a}{b}\right)\right. \\
& \left.+{ }_{2} F_{1}\left(2,1 ; \alpha+2 ; \frac{1}{2}\left(1-\frac{a}{b}\right)\right)\right], \\
C_{2}(\alpha ; a, b)= & \frac{b^{-2}}{\alpha+2}\left[{ }_{2} F_{1}\left(2, \alpha+2 ; \alpha+3 ; 1-\frac{a}{b}\right)-\frac{1}{\alpha+1}{ }_{2} F_{1}\left(2,2 ; \alpha+3 ; 1-\frac{a}{b}\right)\right. \\
& \left.+\frac{1}{2(\alpha+1)}{ }_{2} F_{1}\left(2,1 ; \alpha+3 ; \frac{1}{2}\left(1-\frac{a}{b}\right)\right)\right], \\
C_{3}(\alpha ; a, b)= & \frac{b^{-2}}{\alpha+1}\left[\frac{1}{\alpha+1}{ }_{2} F_{1}\left(2, \alpha+1 ; \alpha+3 ; 1-\frac{a}{b}\right)-{ }_{2} F_{1}\left(2,1 ; \alpha+3 ; 1-\frac{a}{b}\right)\right. \\
& \left.+{ }_{2} F_{1}\left(2,1 ; \alpha+3 ; \frac{1}{2}\left(1-\frac{a}{b}\right)\right)\right],
\end{aligned}
$$

and $0<\alpha \leq 1$.

Theorem 7 ([10]) Let $f: I \subseteq(0, \infty) \rightarrow \mathbb{R}$ be a differentiable function on $I^{\circ}$ such that $f^{\prime} \in$ $L([a, b])$, where $a, b \in I$ with $a<b$. If $\left|f^{\prime}\right|^{q}$ is harmonically convex function on $[a, b]$ for some 
fixed $q>1$, then we have the following inequality for the fractional integrals:

$$
\begin{aligned}
& \left|I_{f}(g ; \alpha, a, b)\right| \\
& \quad \leq \frac{a(b-a)}{2 b}\left(\frac{1}{\alpha p+1}\right)^{\frac{1}{p}}\left(\frac{\left|f^{\prime}(b)\right|^{q}+\left|f^{\prime}(a)\right|^{q}}{2}\right)^{\frac{1}{q}} \\
& \quad \times\left[{ }_{2} F_{1}^{\frac{1}{p}}\left(2 p, 1 ; \alpha p+2 ; 1-\frac{a}{b}\right)+{ }_{2} F_{1}\left(2 p, \alpha p+1 ; \alpha p+2 ; 1-\frac{a}{b}\right)\right],
\end{aligned}
$$

where $\frac{1}{p}+\frac{1}{q}=1$.

Theorem 8 ([10]) Let $f: I \subseteq(0, \infty) \rightarrow \mathbb{R}$ be a differentiable function on $I^{\circ}$ such that $f^{\prime} \in$ $L([a, b])$, where $a, b \in I$ with $a<b$. If $\left|f^{\prime}\right|^{q}$ is harmonically convex function on $[a, b]$ for some fixed $q>1$, then we have the following inequality for the fractional integrals:

$$
\left|I_{f}(g ; \alpha, a, b)\right| \leq \frac{b-a}{2(a b)^{1-\frac{1}{p}}} L_{2 p-2}^{2-\frac{2}{p}}(a, b)\left(\frac{1}{\alpha q+1}\right)^{\frac{1}{q}}\left(\frac{\left|f^{\prime}(b)\right|^{q}+\left|f^{\prime}(a)\right|^{q}}{2}\right)^{\frac{1}{q}},
$$

where $\frac{1}{p}+\frac{1}{q}=1$ and $L_{2 p-2}(a, b)=\left(\frac{b^{2 p-1}-a^{2 p-1}}{(2 p-1)(b-a)}\right)^{\frac{1}{(2 p-2)}}$ is the $2 p-2$-Logarithmic mean.

Theorem 9 ([10]) Let $f: I \subseteq(0, \infty) \rightarrow \mathbb{R}$ be a differentiable function on $I^{\circ}$ such that $f^{\prime} \in$ $L([a, b])$, where $a, b \in I$ with $a<b$. If $\left|f^{\prime}\right|^{q}$ is a harmonically convex function on $[a, b]$ for some fixed $q>1$, then we have the following inequality for the fractional integrals:

$$
\begin{aligned}
\left|I_{f}(g ; \alpha, a, b)\right| \leq & \frac{a(b-a)}{2 b}\left(\frac{1}{\alpha p+1}\right)^{\frac{1}{p}} \\
& \times\left(\frac{{ }_{2} F_{1}\left(2 q, 2 ; 3 ; 1-\frac{a}{b}\right)\left|f^{\prime}(b)\right|^{q}+{ }_{2} F_{1}\left(2 q, 1 ; 3 ; 1-\frac{a}{b}\right)\left|f^{\prime}(a)\right|^{q}}{2}\right)^{\frac{1}{q}},
\end{aligned}
$$

where $\frac{1}{p}+\frac{1}{q}=1$.

For some similar studies with this work of harmonically convex functions, see ([2, 3, 13$15,18])$.

Now we recall the definition of left- and right-sided generalized fractional integrals given by Sarikaya and Ertuğral in [19] as follows:

$$
\begin{aligned}
& { }_{a+} I_{\varphi} f(x)=\int_{a}^{x} \frac{\varphi(x-t)}{x-t} f(t) d t, \quad x>a, \\
& { }_{b-} I_{\varphi} f(x)=\int_{x}^{b} \frac{\varphi(t-x)}{t-x} f(t) d t, \quad x<b,
\end{aligned}
$$

respectively, where the function $\varphi:[0, \infty) \rightarrow[0, \infty)$ satisfies $\int_{0}^{1} \frac{\varphi(t)}{t} d t<\infty$. For details of the generalized fractional integrals see [19].

Some of the special cases of these generalized fractional operators are given as follows.

Remark 1 If we choose $\varphi(t)=t, \varphi(t)=\frac{1}{\Gamma(\alpha)} t^{\alpha}, \varphi(t)=\frac{1}{k \Gamma_{k}(\alpha)} t^{\frac{\alpha}{k}}, k>0, \varphi(t)=t(x-t)^{\alpha-1}$ and $\varphi(t)=\frac{t}{\alpha} \exp \left(-\frac{1-\alpha}{\alpha} t\right), \alpha \in(0,1)$, then we obtain the classical Riemann integral, the 
Riemann-Liouville fractional integral, the $k$-Riemann-Liouville fractional [16], conformable fractional integrals [12] and fractional integral operators with exponential kernel [1], respectively.

The main aim of this paper is to establish inequalities of Hermite-Hadamard type for harmonically convex functions using generalized fractional integrals. Some applications of the results presented in this paper are also obtained.

\section{Main results}

For brevity, throughout in this paper the following notations are used:

$$
T_{f, \Lambda}(g ; a, b)=\frac{f(a)+f(b)}{2}-\frac{1}{2 \Lambda(1)}\left[\frac{1}{a}-I_{\varphi}(f \circ g)\left(\frac{1}{b}\right)+{ }_{\frac{1}{b}}+I_{\varphi}(f \circ g)\left(\frac{1}{a}\right)\right],
$$

where

$$
g(x)=\frac{1}{x}, \quad \Lambda(x)=\int_{0}^{x} \frac{\varphi\left(\frac{(b-a)}{a b} t\right)}{t} d t<+\infty .
$$

We start with the following result.

Theorem 10 Let $f: I \subseteq(0,+\infty) \rightarrow \mathbb{R}$ be a function such that $f \in L([a, b])$. If $f$ is harmonically convex function on $[a, b]$, then the following inequalities hold for the generalized fractional integrals:

$$
f\left(\frac{2 a b}{a+b}\right) \leq \frac{1}{2 \Lambda(1)}\left[\frac{1}{a}-I_{\varphi}(f \circ g)\left(\frac{1}{b}\right)+{ }_{\frac{1}{b}+} I_{\varphi}(f \circ g)\left(\frac{1}{a}\right)\right] \leq \frac{f(a)+f(b)}{2} .
$$

Proof Since $f$ is harmonically convex function on $[a, b]$, we have the following inequality:

$$
f\left(\frac{2 x y}{x+y}\right) \leq \frac{f(x)+f(y)}{2} .
$$

By changing the variables $x=\frac{a b}{t b+(1-t) a}$ and $y=\frac{a b}{t a+(1-t) b}$, the inequality (2.4) becomes

$$
f\left(\frac{2 a b}{a+b}\right) \leq \frac{1}{2}\left[f\left(\frac{a b}{t b+(1-t) a}\right)+f\left(\frac{a b}{t a+(1-t) b}\right)\right] .
$$

Multiplying (2.5) with $\frac{\varphi\left(\frac{(b-a)}{a b} t\right)}{t}$ on both sides and integrating the resulting inequality with respect to $t$ over $[0,1]$, we have

$$
\begin{aligned}
\int_{0}^{1} f\left(\frac{2 a b}{a+b}\right) d t \leq & \frac{1}{2 \Lambda(1)}\left[\int_{0}^{1} \frac{\varphi\left(\frac{(b-a)}{a b} t\right)}{t} f\left(\frac{a b}{t b+(1-t) a}\right) d t\right. \\
& \left.+\int_{0}^{1} \frac{\varphi\left(\frac{(b-a)}{a b} t\right)}{t} f\left(\frac{a b}{t a+(1-t) b}\right) d t\right] \\
= & \frac{1}{2 \Lambda(1)}\left[\frac{1}{a}-I_{\varphi}(f \circ g)\left(\frac{1}{b}\right)+{ }_{\frac{1}{b}+} I_{\varphi}(f \circ g)\left(\frac{1}{a}\right)\right],
\end{aligned}
$$

which is first inequality of our desired result (2.3). 
To prove the second inequality of (2.3), note that $f$ is harmonically convex function and hence the following inequalities hold for $t \in[0,1]$ :

$$
\begin{aligned}
& f\left(\frac{a b}{t b+(1-t) a}\right) \leq t f(a)+(1-t) f(b), \\
& f\left(\frac{a b}{t a+(1-t) b}\right) \leq t f(b)+(1-t) f(a) .
\end{aligned}
$$

By adding (2.6) and (2.7), we have

$$
f\left(\frac{a b}{t b+(1-t) a}\right)+f\left(\frac{a b}{t a+(1-t) b}\right) \leq f(a)+f(b) .
$$

On multiplying the both sides of (2.8) by $\frac{\varphi\left(\frac{(b-a)}{a b} t\right)}{t}$ and integrating the result with respect to $t$ on $[0,1]$, we obtain

$$
\begin{aligned}
& \int_{0}^{1} \frac{\varphi\left(\frac{(b-a)}{a b} t\right)}{t} f\left(\frac{a b}{t b+(1-t) a}\right) d t+\int_{0}^{1} \frac{\varphi\left(\frac{(b-a)}{a b} t\right)}{t} f\left(\frac{a b}{t a+(1-t) b}\right) d t \\
& \quad \leq \Lambda(1)[f(a)+f(b)],
\end{aligned}
$$

by changing the variables $x=\frac{a b}{t b+(1-t) a}$ and $y=\frac{a b}{t a+(1-t) b}$, the inequality (2.9) becomes

$$
\left[\frac{1}{a}+I_{\varphi}(f \circ g)\left(\frac{1}{b}\right)+{ }_{\frac{1}{b}}-I_{\varphi}(f \circ g)\left(\frac{1}{a}\right)\right] \leq \Lambda(1)[f(a)+f(b)] .
$$

Hence we have the proof of Theorem 10.

Remark 2 Under the assumptions of Theorem 10, if we take $\varphi(t)=t$, then inequalities (2.3) reduce to inequalities (1.3).

Remark 3 Under the assumptions of Theorem 10, if we define $\varphi(t)=\frac{t^{\alpha}}{\Gamma(\alpha)}$, then inequalities (2.3) reduce to inequalities (1.7).

Corollary 1 Under the assumptions of Theorem 10, if we take $\varphi(t)=\frac{t^{\frac{\alpha}{k}}}{k \Gamma_{k}(\alpha)}$, then we have

$$
\begin{aligned}
f\left(\frac{2 a b}{a+b}\right) & \leq \frac{\Gamma_{k}(\alpha+k)}{2}\left(\frac{a b}{b-a}\right)^{\frac{\alpha}{k}}\left\{I_{\frac{1}{a}-}^{\alpha, k}(f \circ g)\left(\frac{1}{b}\right)+I_{\frac{1}{b}+}^{\alpha, k}(f \circ g)\left(\frac{1}{a}\right)\right\} \\
& \leq \frac{f(a)+f(b)}{2} .
\end{aligned}
$$

Corollary 2 Under the assumptions of Theorem 10, if we take $\varphi(t)=t(b-t)^{\alpha-1}$, then we obtain the following inequalities:

$$
f\left(\frac{2 a b}{a+b}\right) \leq \frac{1}{2 \Lambda^{*}(1)}\left[\frac{1}{a}-I_{\varphi}(f \circ g)\left(\frac{1}{b}\right)+{ }_{\frac{1}{b}+} I_{\varphi}(f \circ g)\left(\frac{1}{a}\right)\right] \leq \frac{f(a)+f(b)}{2},
$$

where

$$
\Lambda^{*}(1)=\frac{b^{\alpha}-\left(b-\frac{b-a}{a b}\right)^{\alpha}}{\alpha} .
$$


Corollary 3 Under the assumptions of Theorem 10, taking $\varphi(t)=\frac{t}{\alpha} \exp \left(-\frac{1-\alpha}{\alpha} t\right), \alpha \in(0,1)$, we obtain

$$
f\left(\frac{2 a b}{a+b}\right) \leq \frac{1}{2 \Lambda^{* *}(1)}\left[\frac{1}{a}-I_{\varphi}(f \circ g)\left(\frac{1}{b}\right)+{ }_{\frac{1}{b}+} I_{\varphi}(f \circ g)\left(\frac{1}{a}\right)\right] \leq \frac{f(a)+f(b)}{2},
$$

where

$$
\Lambda^{* *}(1)=\frac{1-\exp \left(\frac{(\alpha-1)(b-a)}{\alpha a b}\right)}{1-\alpha} .
$$

The next lemma is very crucial in the proof of our next results.

Lemma 4 Let $f: I \subseteq(0,+\infty) \rightarrow \mathbb{R}$ be a differentiable function on $I^{\circ}$ such that $f^{\prime} \in L([a, b])$, where $a, b \in I$ with $a<b$. Then the following identity holds for the generalized fractional integrals:

$$
T_{f, \Lambda}(g ; a, b)=\frac{1}{2 \Lambda(1)} \int_{0}^{1} \frac{[\Lambda(1-t)-\Lambda(t)]}{(t b+(1-t) a)^{2}} f^{\prime}\left(\frac{a b}{t b+(1-t) a}\right) d t .
$$

Proof Denote

$$
T_{f, \Lambda}(g ; a, b)=\frac{1}{2 \Lambda(1)}\left[T_{f, \Lambda}^{(1)}(g ; a, b)-T_{f, \Lambda}^{(2)}(g ; a, b)\right],
$$

where

$$
T_{f, \Lambda}^{(1)}(g ; a, b)=\int_{0}^{1} \frac{\Lambda(1-t)}{(t b+(1-t) a)^{2}} f^{\prime}\left(\frac{a b}{t b+(1-t) a}\right) d t
$$

and

$$
T_{f, \Lambda}^{(2)}(g ; a, b)=\int_{0}^{1} \frac{\Lambda(t)}{(t b+(1-t) a)^{2}} f^{\prime}\left(\frac{a b}{t b+(1-t) a}\right) d t .
$$

Integrating (2.15) by parts, we have

$$
\begin{aligned}
T_{f, \Lambda}^{(1)}(g ; a, b)= & -\left.\Lambda(1-t) f\left(\frac{a b}{t b+(1-t) a}\right)\right|_{0} ^{1} \\
& -\int_{0}^{1} \frac{\varphi\left(\frac{(b-a)}{a b}(1-t)\right)}{1-t} f\left(\frac{a b}{t b+(1-t) a}\right) d t \\
= & \Lambda(1) f(b)-{ }_{\frac{1}{b}}+I_{\varphi}(f \circ g)\left(\frac{1}{a}\right) .
\end{aligned}
$$

Similarly, using (2.16), we get

$$
T_{f, \Lambda}^{(2)}(g ; a, b)=-\Lambda(1) f(a)+{ }_{\frac{1}{a}}-I_{\varphi}(f \circ g)\left(\frac{1}{b}\right) .
$$

Substituting (2.17) and (2.18) in (2.14), we obtain (2.13) which completes the proof of Lemma 4. 
Remark 4 Under the assumptions of Lemma 4, if we take $\varphi(t)=t$, the identity (2.13) reduces to (1.4).

Remark 5 Under the assumptions of Lemma 4, taking $\varphi(t)=\frac{t^{\alpha}}{\Gamma(\alpha)}$, the identity (2.13) reduces to identity (1.8).

Theorem 11 Let $f: I \subseteq(0,+\infty) \rightarrow \mathbb{R}$ be a differentiable function on $I^{\circ}$ such that $f^{\prime} \in$ $L([a, b])$, where $a, b \in I$ with $a<b$. If $\left|f^{\prime}\right|^{q}$ is harmonically convex on $[a, b]$ for some $q \geq 1$, then the following inequality holds for the generalized fractional integrals:

$$
\left|T_{f, \Lambda}(g ; a, b)\right| \leq B_{1, \Lambda}^{1-\frac{1}{q}}(a, b)\left(B_{2, \Lambda}(a, b)\left|f^{\prime}(a)\right|^{q}+B_{3, \Lambda}(a, b)\left|f^{\prime}(b)\right|^{q}\right)^{\frac{1}{q}}
$$

where

$$
\begin{aligned}
& B_{1, \Lambda}(a, b)=\int_{0}^{1} \frac{[\Lambda(1-t)-\Lambda(t)]}{(t b+(1-t) a)^{2}} d t, \\
& B_{2, \Lambda}(a, b)=\int_{0}^{1} \frac{[\Lambda(1-t)-\Lambda(t)]}{(t b+(1-t) a)^{2}} t d t \\
& B_{3, \Lambda}(a, b)=\int_{0}^{1} \frac{[\Lambda(1-t)-\Lambda(t)]}{(t b+(1-t) a)^{2}}(1-t) d t .
\end{aligned}
$$

Proof From Lemma 4 and the well-known power mean inequality, we have

$$
\begin{aligned}
\left|T_{f, \Lambda}(g ; a, b)\right| \leq & \int_{0}^{1} \frac{|[\Lambda(1-t)-\Lambda(t)]|}{(t b+(1-t) a)^{2}}\left|f^{\prime}\left(\frac{a b}{t b+(1-t) a}\right)\right| d t \\
\leq & \left(\int_{0}^{1} \frac{|[\Lambda(1-t)-\Lambda(t)]|}{(t b+(1-t) a)^{2}} d t\right)^{1-\frac{1}{q}} \\
& \times\left(\int_{0}^{1} \frac{|[\Lambda(1-t)-\Lambda(t)]|}{(t b+(1-t) a)^{2}}\left|f^{\prime}\left(\frac{a b}{t b+(1-t) a}\right)\right|^{q} d t\right)^{\frac{1}{q}} \\
\leq & \left(\int_{0}^{1} \frac{[\Lambda(1-t)-\Lambda(t)]}{(t b+(1-t) a)^{2}} d t\right)^{1-\frac{1}{q}} \\
& \times\left(\int_{0}^{1} \frac{[\Lambda(1-t)-\Lambda(t)]}{(t b+(1-t) a)^{2}}\left[t\left|f^{\prime}(a)\right|^{q}+(1-t)\left|f^{\prime}(b)\right|^{q}\right] d t\right)^{\frac{1}{q}} \\
= & \left(\int_{0}^{1} \frac{[\Lambda(1-t)-\Lambda(t)]}{(t b+(1-t) a)^{2}} d t\right)^{1-\frac{1}{q}} \\
& \times\left(\int_{0}^{1} \frac{[\Lambda(1-t)-\Lambda(t)]}{(t b+(1-t) a)^{2}} t\left|f^{\prime}(a)\right|^{q} d t\right. \\
& \left.+\int_{0}^{1} \frac{[\Lambda(1-t)-\Lambda(t)]}{(t b+(1-t) a)^{2}}(1-t)\left|f^{\prime}(b)\right|^{q} d t\right)^{\frac{1}{q}} \\
= & B_{1, \Lambda}^{1-\frac{1}{q}}(a, b)\left(B_{2, \Lambda}(a, b)\left|f^{\prime}(a)\right|+B_{3, \Lambda}(a, b)\left|f^{\prime}(b)\right|\right)^{\frac{1}{q}},
\end{aligned}
$$

which is our required inequality (2.19). 
Remark 6 Under the assumptions of Theorem 11, if we define $\varphi(t)=t$, and $\varphi(t)=\frac{t^{\alpha}}{\Gamma(\alpha)}$, then inequality (2.19) reduces to the inequalities (1.5), (1.9), respectively.

Remark 7 Under the assumptions of Theorem 11, taking $\varphi(t)=\frac{t^{\alpha}}{\Gamma(\alpha)}$ and using Lemma 2, the inequality (2.19) reduces to the inequality (1.10).

Corollary 4 Under the assumptions of Theorem 11, taking $\varphi(t)=\frac{t^{\frac{\alpha}{k}}}{k \Gamma_{k}(\alpha)}$, the inequality

$$
\left|T_{f, \Lambda}(g ; a, b)\right| \leq B_{1, \Lambda}^{1-\frac{1}{q}}(a, b)\left(B_{2, \Lambda}(a, b)\left|f^{\prime}(a)\right|^{q}+B_{3, \Lambda}(a, b)\left|f^{\prime}(b)\right|^{q}\right)^{\frac{1}{q}}
$$

is obtained, where

$$
\Lambda(t)=\frac{\left(\frac{(b-a) t}{a b}\right)^{\frac{\alpha}{k}}}{\Gamma_{k}(\alpha+k)} .
$$

Corollary 5 Under the assumptions of Theorem 11, if we take $\varphi(t)=t(b-t)^{\alpha-1}$, then we have

$$
\left|T_{f, \Lambda^{*}}(g ; a, b)\right| \leq B_{1, \Lambda^{*}}^{1-\frac{1}{q}}(a, b)\left(B_{2, \Lambda^{*}}(a, b)\left|f^{\prime}(a)\right|^{q}+B_{3, \Lambda^{*}}(a, b)\left|f^{\prime}(b)\right|^{q}\right)^{\frac{1}{q}},
$$

where

$$
\Lambda^{*}(t)=\frac{b^{\alpha}-\left(b-\frac{(b-a) t}{a b}\right)^{\alpha}}{\alpha} .
$$

Corollary 6 Under the assumptions of Theorem 11, if we choose $\varphi(t)=\frac{t}{\alpha} \exp \left(-\frac{1-\alpha}{\alpha} t\right), \alpha \in$ $(0,1)$, then we have

$$
\left|T_{f, \Lambda^{* *}}(g ; a, b)\right| \leq B_{1, \Lambda^{* *}}^{1-\frac{1}{q}}(a, b)\left(B_{2, \Lambda^{* *}}(a, b)\left|f^{\prime}(a)\right|^{q}+B_{3, \Lambda^{* *}}(a, b)\left|f^{\prime}(b)\right|^{q}\right)^{\frac{1}{q}},
$$

where

$$
\Lambda^{* *}(t)=\frac{1-\exp \left(\frac{(\alpha-1)(b-a) t}{\alpha a b}\right)}{1-\alpha} .
$$

Theorem 12 Let $f: I \subseteq(0,+\infty) \rightarrow \mathbb{R}$ be a differentiable function on $I^{\circ}$ such that $f^{\prime} \in$ $L([a, b])$, where $a, b \in I^{\circ}$ with $a<b$. If $\left|f^{\prime}\right|^{q}$ is harmonically convex on $[a, b]$ for some fixed $q>1$, then the following inequality for generalized fractional integrals holds:

$$
\left|T_{f, \Lambda}(g ; a, b)\right| \leq\left(B_{4, \Lambda}^{\frac{1}{p}}(a, b)+B_{5, \Lambda}^{\frac{1}{p}}(a, b)\right)\left(\frac{\left|f^{\prime}\right|^{q}+\left|f^{\prime}\right|^{q}}{2}\right)^{\frac{1}{q}},
$$

where

$$
\begin{aligned}
& B_{4, \Lambda}(a, b)=\int_{0}^{1} \frac{(\Lambda(1-t))^{p}}{(t b+(1-t) a)^{2 p}} d t, \\
& B_{5, \Lambda}(a, b)=\int_{0}^{1} \frac{(\Lambda(t))^{p}}{(t b+(1-t) a)^{2 p}} d t,
\end{aligned}
$$

and $\frac{1}{p}+\frac{1}{q}=1$. 
Proof It follows from Lemma 4 and the Hölder inequality that

$$
\begin{aligned}
\left|T_{f, \Lambda}(g ; a, b)\right| \leq & \int_{0}^{1} \frac{|[\Lambda(1-t)-\Lambda(t)]|}{(t b+(1-t) a)^{2}}\left|f^{\prime}\left(\frac{a b}{t b+(1-t) a}\right)\right| d t \\
= & \int_{0}^{1} \frac{\Lambda(1-t)}{(t b+(1-t) a)^{2}}\left|f^{\prime}\left(\frac{a b}{t b+(1-t) a}\right)\right| d t \\
& +\int_{0}^{1} \frac{\Lambda(t)}{(t b+(1-t) a)^{2}}\left|f^{\prime}\left(\frac{a b}{t b+(1-t) a}\right)\right| d t \\
\leq & \left(\int_{0}^{1} \frac{(\Lambda(1-t))^{p}}{(t b+(1-t) a)^{2 p}} d t\right)^{\frac{1}{p}}\left(\int_{0}^{1}\left|f^{\prime}\left(\frac{a b}{t b+(1-t) a}\right)\right|^{q} d t\right)^{\frac{1}{q}} \\
& +\left(\int_{0}^{1} \frac{(\Lambda(t))^{p}}{(t b+(1-t) a)^{2 p}} d t\right)^{\frac{1}{p}}\left(\int_{0}^{1}\left|f^{\prime}\left(\frac{a b}{t b+(1-t) a}\right)\right|^{q} d t\right)^{\frac{1}{q}} \\
\leq & \left(\int_{0}^{1} \frac{(\Lambda(1-t))^{p}}{(t b+(1-t) a)^{2 p}} d t\right)^{\frac{1}{p}}\left(\int_{0}^{1}\left(t\left|f^{\prime}(a)\right|^{q}+(1-t)\left|f^{\prime}(b)\right|^{q}\right) d t\right)^{\frac{1}{q}} \\
& \times\left(\int_{0}^{1} \frac{(\Lambda(t))^{p}}{(t b+(1-t) a)^{2 p}} d t\right)^{\frac{1}{p}}\left(\int_{0}^{1}\left(t\left|f^{\prime}(a)\right|^{q}+(1-t)\left|f^{\prime}(b)\right|^{q}\right) d t\right)^{\frac{1}{q}} \\
= & \left(B_{4, \Lambda}^{\frac{1}{p}}(a, b)+B_{5, \Lambda}^{\frac{1}{p}}(a, b)\right)\left(\frac{\left|f^{\prime q}+\right| f^{\prime q}}{2}\right) \\
\frac{1}{q} &
\end{aligned}
$$

This completes the proof of Theorem 12.

Remark 8 Under the assumptions of Theorem 12, taking $\varphi(t)=t$ and $\varphi(t)=\frac{t^{\alpha}}{\Gamma(\alpha)}$, the inequality (2.23) reduces to the inequalities (1.6) and (1.11), respectively.

Remark 9 Under the assumptions of Theorem 12, if we take $\varphi(t)=\frac{t^{\alpha}}{\Gamma(\alpha)}$ and use the Lemma 2, then the inequality (2.23) reduces to the inequalities (1.12) and (1.13).

Corollary 7 Under the assumptions of Theorem 12, $\varphi(t)=\frac{t^{\frac{\alpha}{k}}}{k \Gamma_{k}(\alpha)}$ gives

$$
\left|T_{f, \Lambda}(g ; a, b)\right| \leq\left(B_{4, \Lambda}^{\frac{1}{p}}(a, b)+B_{5, \Lambda}^{\frac{1}{p}}(a, b)\right)\left(\frac{\left|f^{\prime}\right|^{q}+\left|f^{\prime}\right|^{q}}{2}\right)^{\frac{1}{q}}
$$

where $\Lambda(t)$ is defined in Corollary 4.

Corollary 8 Under the assumptions of Theorem 12, if we take $\varphi(t)=t(b-t)^{\alpha-1}$, then we obtain

$$
\left|T_{f, \Lambda^{*}}(g ; a, b)\right| \leq\left(B_{4, \Lambda^{*}}^{\frac{1}{p}}(a, b)+B_{5, \Lambda^{*}}^{\frac{1}{p}}(a, b)\right)\left(\frac{\left|f^{\prime}\right|^{q}+\left|f^{\prime}\right|^{q}}{2}\right)^{\frac{1}{q}}
$$

where $\Lambda^{*}(t)$ is defined as in Corollary 5 . 
Corollary 9 Under the assumptions of Theorem 12, if we set $\varphi(t)=\frac{t}{\alpha} \exp \left(-\frac{1-\alpha}{\alpha} t\right), \alpha \in(0,1)$, then we have the following inequality:

$$
\left|T_{f, \Lambda^{* *}}(g ; a, b)\right| \leq\left(B_{4, \Lambda^{* *}}^{\frac{1}{p}}(a, b)+B_{5, \Lambda^{* *}}^{\frac{1}{p}}(a, b)\right)\left(\frac{\left|f^{\prime}\right|^{q}+\left|f^{\prime}\right|^{q}}{2}\right)^{\frac{1}{q}},
$$

where $\Lambda^{* *}(t)$ is defined in Corollary 6.

\section{Applications to special means}

Let us consider some means for positive real numbers $\ell_{1}$ and $\ell_{2}$, where $\ell_{1}<\ell_{2}$, as follows:

(1) The arithmetic mean:

$$
A\left(\ell_{1}, \ell_{2}\right)=\frac{\ell_{1}+\ell_{2}}{2} .
$$

(2) The geometric mean:

$$
G\left(\ell_{1}, \ell_{2}\right)=\sqrt{\ell_{1} \ell_{2}}
$$

(3) The generalized log-mean:

$$
L_{p}\left(\ell_{1}, \ell_{2}\right)=\left[\frac{\ell_{2}^{p+1}-\ell_{1}^{p+1}}{(p+1)\left(\ell_{2}-\ell_{1}\right)}\right]^{\frac{1}{p}} ; \quad p \in \mathbb{R} \backslash\{-1,0\} .
$$

Proposition 1 Under the assumptions of Theorem 11, take $\varphi(t)=t$ to obtain

$$
\begin{aligned}
\left|A\left(\ell_{1}^{p+2}, \ell_{2}^{p+2}\right)-G^{2}\left(\ell_{1}, \ell_{2}\right) L_{p}^{p}\left(\ell_{1}, \ell_{2}\right)\right| \leq & 2^{\frac{1-q}{q}}(p+2) G^{2}\left(\ell_{1}, \ell_{2}\right)\left(\ell_{2}-\ell_{1}\right) \lambda_{1}^{1-\frac{1}{q}} \\
& \times \sqrt[q]{A\left(\lambda_{2} \ell_{1}^{q(p+1)}, \lambda_{3} \ell_{2}^{q(p+1)}\right)},
\end{aligned}
$$

where $\lambda_{1}, \lambda_{2}$ and $\lambda_{3}$ are given in Theorem 2 .

Proof Taking $f(x)=x^{p+2}$, where $x>0$ and $p \in(-1,+\infty) \backslash\{0\}$ in Theorem 11, (3.1) is obtained.

Proposition 2 Under the assumptions of Theorem 12, $\varphi(t)=t$ gives

$$
\begin{aligned}
& \left|A\left(\ell_{1}^{s+2}, \ell_{2}^{s+2}\right)-G^{2}\left(\ell_{1}, \ell_{2}\right) L_{s}^{s}\left(\ell_{1}, \ell_{2}\right)\right| \\
& \quad \leq 2^{\frac{1-q}{q}} \frac{(s+2)}{\sqrt[p]{p+1}} G^{2}\left(\ell_{1}, \ell_{2}\right)\left(\ell_{2}-\ell_{1}\right) \sqrt[q]{A\left(\mu_{1} \ell_{1}^{q(s+1)}, \mu_{2} \ell_{2}^{q(s+1)}\right)},
\end{aligned}
$$

where $\mu_{1}$ and $\mu_{2}$ are the same as in Theorem 3.

Proof Taking $f(x)=x^{s+2}$, where $x>0$ and $s \in(-1,+\infty) \backslash\{0\}$ in Theorem 12, (3.2) is obtained.

Remark 10 Under the assumptions of Theorems 11 and 12, for appropriate choices of the functions such as

$$
\varphi(t)=\frac{t^{\alpha}}{\Gamma(\alpha)} ; \frac{t^{\frac{\alpha}{k}}}{k \Gamma_{k}(\alpha)} ; t(b-t)^{\alpha-1} \text { and } \frac{t}{\alpha} \exp \left(-\frac{1-\alpha}{\alpha} t\right)
$$


and for harmonically convex function $f(x)=x^{p+2}$, where $x>0$ and $p \in(-1,+\infty) \backslash\{0\} ; x^{2} \ln x$, where $x>0$, we obtain some new interesting inequalities using the special means. The details are left to the reader.

\section{Conclusion}

In this paper, we established inequalities of Hermite-Hadamard type for harmonically convex functions using generalized fractional integrals. Some special cases are provided as well. Finally, some application to special means are given. The results of the present paper can be applied in convex analysis, optimization and also different areas of pure and applied sciences.

\section{Acknowledgements}

We thankful to editor and referees for their careful reading and valuable suggestions to make the article better readable.

\section{Funding}

This work was supported in part by Special Soft Science Research Projects of Technological Innovation in Hubei Province (2019ADC46), the Fundamental Research Funds for Central Universities (2019B44914), Key Projects of Education Commission of Hubie Province of China (D20192501), the Natural Science Foundation of Jiangsu Province (BK20180500), the National Key Research and Development Program of China (2018YFC1508100) and partially supported by the National Natural Science Foundation of China (11971241).

\section{Availability of data and materials}

Data sharing not applicable to this paper as no data sets were generated or analyzed during the current study.

\section{Competing interests}

It is declared that the authors have no competing interests.

\section{Authors' contributions}

The study was carried out in collaboration of all authors. All authors read and approved the final manuscript.

\section{Author details}

${ }^{1}$ College of Science, Hohai University, Nanjing, P.R. China. ${ }^{2}$ School of Mathematics and Statistics, Hubei Normal University, Huangshi, P.R. China. ${ }^{3}$ Jiangsu Key Laboratory for NSLSCS, School of Mathematical Sciences, Nanjing Normal University, Nanjing, China. ${ }^{4}$ Department of Mathematics, Faculty of Technical Sciences, University of Ismail Qemali, Vlora, Albania.

${ }^{5}$ Department of Mathematics, Faculty of Science and Arts, Düzce University, Düzce, Turkey.

\section{Publisher's Note}

Springer Nature remains neutral with regard to jurisdictional claims in published maps and institutional affiliations.

Received: 2 January 2020 Accepted: 17 March 2020 Published online: 26 March 2020

\section{References}

1. Ahmad, B., Alsaedi, A., Kirane, M., Torebek, B.T.: Hermite-Hadamard, Hermite-Hadamard-Fejer, Dragomir-Agarwal and Pachpatte type inequalities for convex functions via fractional integrals. J. Comput. Appl. Math. 353, 120-129 (2019)

2. Awan, M.U., Noor, M.A., Minhai, M.V., Noor, K.I. Inequalities via harmonic functions: conformable fractional calculus approach. J. Math. Inequal. 12(1), 143-153 (2018)

3. Dragomir, S.S.: Inequalities of Hermite-Hadamard type for HA-convex functions. Moroccan J. Pure Appl. Anal. 3(1), 83-101 (2017)

4. Dragomir, S.S., Pearce, C.E.M.: Selected Topics on Hermite-Hadamard Inequalities and Applications. RGMIA Monographs, Victoria University (2000)

5. Iş̧can, I.: New estimates on generalization of some integral inequalities for $(\alpha, m)$-convex functions. Contemp. Anal. Appl. Math. 1(2), 253-264 (2013)

6. Işcan, I: New estimates on generalization of some integral inequalities for $s$-convex functions and their applications. Int. J. Pure Appl. Math. 86(4), 727-746 (2013)

7. İscan, I.: New general integral inequalities for quasi-geometrically convex functions via fractional integrals. J. Inequal. Appl. 2013(491), 15 (2013)

8. Iş̧an, l.: Hermite-Hadamard type inequalities for harmonically convex functions. Hacet. J. Math. Stat. 43(6), 935-942 (2014)

9. İşcan, l.: Generalization of different type integral inequalities for $s$-convex functions via fractional integrals. Appl. Anal. 93(9), 1846-1862 (2014)

10. Işcan, I., Wu, S.: Hermite-Hadamard type inequalities for harmonically convex functions via fractional integrals. Appl. Math. Comput. 238, 237-244 (2014)

11. Kavurmac, H., Özdemir, M.E., Avc, M.: New Ostrowski type inequalities for $m$-convex functions and applications. Hacet. J. Math. Stat. 40(2), 135-145 (2011) 
12. Khalil, R., Al Horani, M., Yousef, A., Sababheh, M.: A new definition of fractional derivative. J. Comput. Appl. Math. 264, 65-70 (2014)

13. Kunt, M., Iscan, I.: Hermite-Hadamard type inequalities for harmonically $(\alpha, m)$-convex functions by using fractional integrals. Konuralp J. Math. 5(1), 201-213 (2017)

14. Kunt, M., Iscan, I., Yazici, N.: Hermite-Hadamard type inequalities for product of harmonically convex functions via Riemann-Liouvile fractional integrals. J. Math. Anal. 7(4), 74-82 (2016)

15. Minhai, M.V., Awan, M.U., Noor, M.A., Noor, K.I. Fractional Hermite-Hadamard inequalities containing generalized Mittag-Leffler functions. J. Inequal. Appl. 2017, 265, 1-13 (2017)

16. Mubeen, S., Habibullah, G.M.: k-Fractional integrals and application. Int. J. Contemp. Math. Sci. 7(2), 89-94 (2012)

17. Pečarić, J.E., Proschan, F., Tong, Y.L.: Convex Functions, Partial Orderings and Statistical Applications. Academic Press, Boston (1992)

18. Şanli, Z:: Some mid point type inequalities for Riemann-Liouville fractional integrals. Appl. Appl. Math. 3, 58-73 (2019)

19. Sarikaya, M.Z., Ertuğral, F.: On the generalized Hermite-Hadamard inequalities, submitted, December 2017, https://www.researchgate.net/publication/321760443

20. Sarikaya, M.Z., Set, E., Yaldiz, H., Basak, N.: Hermite-Hadamard's inequalities for fractional integrals and related fractional inequalities. Math. Comput. Model. 57, 2403-2407 (2013)

21. Set, E.: New inequalities of Ostrowski type for mappings whose derivatives are s-convex in the second sense via fractional integrals. Comput. Math. Appl. 63, 1147-1154 (2012)

22. Set, E., Ozdemir, M.E., Dragomir, S.S.: On Hadamard-type inequalities involving several kinds of convexity. J. Inequal. Appl. 2010, Article ID 286845, 12 (2010). https://doi.org/10.1155/2010/286845

23. Wang, J., Li, X., Feĉkan, M., Zhou, Y.: Hermite-Hadamard-type inequalities for Riemann-Liouville fractional integrals via two kinds of convexity. Appl. Anal. 92(11), 2241-2253 (2012). https://doi.org/10.1080/00036811.2012.727986

24. Wang, J., Zhu, C., Zhou, Y.: New generalized Hermite-Hadamard type inequalities and applications to special means. J. Inequal. Appl. 2013, 325, 15 pp. (2013)

\section{Submit your manuscript to a SpringerOpen ${ }^{\circ}$ journal and benefit from:}

- Convenient online submission

- Rigorous peer review

- Open access: articles freely available online

- High visibility within the field

- Retaining the copyright to your article

Submit your next manuscript at $\gg$ springeropen.com 